\title{
Two-Photon Excitation of trans-Stilbene: Spectroscopy and Dynamics of Electronically Excited States Above $S_{1}$
}

\author{
Amanda L. Houk, Igor L. Zheldakov ${ }^{\dagger}$, Tyler A. Tommey ${ }^{*}$, and Christopher G. Elles* \\ Department of Chemistry, University of Kansas, Lawrence, Kansas 66045
}

\begin{abstract}
The photoisomerization dynamics of trans-stilbene have been well studied in the lowest excited state, but much less is known about the behavior following excitation to higher-lying electronically excited states. This contribution reports a combined study of the spectroscopy and dynamics of two-photon accessible states above $S_{1}$. Two-photon absorption (2PA) measurements using a broadband pump-probe technique reveal distinct bands near 5.1 and $6.4 \mathrm{eV}$. The $2 \mathrm{PA}$

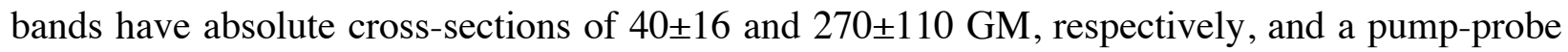
polarization dependence that suggests both of the transitions access $\mathrm{A}_{\mathrm{g}}$-symmetry excited states. Separate transient absorption measurements probe the excited-state dynamics following twophoton excitation into each of the bands using intense pulses of 475- and 380-nm light, respectively. The initially excited states rapidly relax via internal conversion, leading to the formation of an $S_{1}$ excited-state absorption band that is centered near $585 \mathrm{~nm}$ and evolves on a timescale of 1-2 ps due to intramolecular vibrational relaxation. The subsequent evolution of the $\mathrm{S}_{1}$ excited-state absorption is identical to the behavior following direct one-photon excitation of the lowest excited state at $4.0 \mathrm{eV}$. The complementary spectroscopy and dynamics measurements provide new benchmarks for computational studies of the electronic structure and dynamics of this model system on excited states above $S_{1}$. Probing the dynamics of molecules in their higherlying excited states is an important frontier in chemical reaction dynamics.
\end{abstract}

\section{KEYWORDS}

Two-photon absorption, higher excited states, ultrafast spectroscopy, isomerization 


\section{INTRODUCTION}

Probing the ultrafast dynamics of molecules in electronically excited states above $\mathrm{S}_{1}$ represents an important frontier in the field of chemical reaction dynamics that challenges the current limits of both experiment and theory. ${ }^{1}$ Among other complications, the rapidly increasing density of electronic states leads to strong configurational mixing and very short excited-state lifetimes for even the smallest of molecules, as manifest in Kasha's rule. ${ }^{2}$ Nevertheless, probing the behavior of these highly excited systems is important for developing a predictive and general understanding of the non-adiabatic dynamics that govern chemical reactions under a wide range of conditions.

Extensive work over the past 70 years provides significant insight into the photoisomerization dynamics of stilbene, making this compound an ideal prototype for studying excited-state dynamics. ${ }^{3-7}$ Experimental $^{8-18}$ and theoretical ${ }^{19-27}$ studies reveal a mechanism in which torsional rotation around the central $\mathrm{C}=\mathrm{C}$ double bond carries the molecule over a small barrier in the $S_{1}$ excited state before passing through a conical intersection (CI) that returns the molecule to $\mathrm{S}_{0}$. The CI connecting the ground and excited electronic states is located near the minimum of the $S_{1}$ excited-state potential energy surface, where the phenyl rings have perpendicular orientation, but also requires some degree of pyramidalization at one of the ethylene carbon atoms. ${ }^{24,26}$ Passage through the CI results in a roughly 1:1 ratio of cis- and transstilbene. $^{3}$

One-photon excitation of the lowest excited state of trans-stilbene initially accesses a singly excited state with $\pi \pi^{*}$ character. However, the electronic configuration that correlates diabatically with the ground electronic state of the product, and therefore participates in the CI, is a doubly excited state with $\pi^{*} \pi^{*}$ character (becoming $\pi \pi$ in the ground state). ${ }^{20,28}$ This twoelectron excited state of trans-stilbene has been observed previously using two-photon absorption spectroscopy, ${ }^{28-34}$ which is suitable for accessing doubly excited states because of the propensity for two-photon-two-electron interactions. ${ }^{35}$ Two-photon excitation also satisfies the symmetry selection rules for accessing the totally symmetric $\left(\mathrm{A}_{\mathrm{g}}\right) \pi^{*} \pi^{*}$ state, therefore the 
electronic configuration that correlates diabatically with the product ground state is accessible directly via a symmetry-allowed two-photon $(\mathrm{HOMO})^{2} \rightarrow(\mathrm{LUMO})^{2}$ transition from the equilibrium ground state of the trans isomer. Although there have been no direct reports of the excited-state dynamics following two-photon excitation, competing kinetics measurements for both isomers indicate that the cis-trans isomerization process is more efficient following twophoton excitation than for one-photon excitation..$^{36-38}$

Despite the detailed picture that emerges for dynamics on the lowest $\pi \pi^{*}$ excited state of stilbene, and even for the $\pi^{*} \pi^{*}$ excited state, much less is known about the dynamics in the higher-lying excited states, or how excitation to these levels might be used to selectively control the photoisomerization reaction. Recently, Bao and Weber probed the dynamics of cis- and trans-stilbene in the gas phase following excitation of one-photon-accessible excited-states lying roughly $6 \mathrm{eV}$ above the respective ground-state minima, and observed distinctly different dynamics than the well-known torsional isomerization pathway that occurs on $S_{1} \cdot{ }^{16,17,39}$ In particular, coherent oscillations in the higher-lying excited-state $\left(S_{5}\right)$ of trans-stilbene were attributed to an antisymmetric twisting motion of the phenyl groups about the $\mathrm{C}-\mathrm{C}$ single bonds, which suggests that excitation to higher excited states gives rise to different initial motions of trans-stilbene than on the $\mathrm{S}_{1}$ surface. Similarly, Kovalenko and coworkers ${ }^{12}$ probed the solutionphase photoisomerization dynamics of stilbene following one-photon excitation to the second absorption band at $226 \mathrm{~nm}$, from which they observed rapid (sub-100 fs) internal conversion followed by vibrational cooling on the lowest excited state.

The experiments presented in this paper examine the spectroscopy and dynamics of twophoton-accessible excited-states of trans-stilbene in solution. Mutually exclusive selection rules due to the inversion center of trans-stilbene ensure that one- and two-photon excitation access different excited states of the molecule. Here, we present the first continuous 2PA spectrum of trans-stilbene up to $6.5 \mathrm{eV}$, and then compare the excited-state dynamics following one- and two-photon excitation. Our results provide new benchmarks for computational studies of the electronic structure and excited-state dynamics of trans-stilbene above $S_{1}$. 


\section{EXERIMENTAL METHODS}

We use ultrafast pump-probe techniques to examine the spectroscopy and dynamics of trans-stilbene in solution. Briefly, the two-photon absorption (2PA) spectrum is obtained from the wavelength-dependent attenuation of a broadband probe pulse that is temporally and spatially overlapped in the sample with a non-resonant pump pulse, ${ }^{40-44}$ whereas the excited-state dynamics are monitored via the evolution of the transient absorption (TA) spectrum as a function of time following one- or two-photon excitation. ${ }^{47,48}$ In both experiments, pump and probe pulses are derived from the output of a regeneratively amplified Ti:Sapphire laser (Legend Elite, Coherent). Non-linear frequency conversion produces pump pulses that are tunable across the visible-UV, and continuum generation in a $2 \mathrm{~mm} \mathrm{CaF}_{2}$ crystal produces broadband probe pulses covering the range $750-350 \mathrm{~nm}$. We control the relative polarization of the linearly polarized pump and probe light by rotating a $\lambda / 2$ waveplate in the $800 \mathrm{~nm}$ fundamental prior to continuum generation, while also rotating the $\mathrm{CaF}_{2}$ crystal to maintain the polarization purity of the probe beam. ${ }^{45,46}$ After passing through the sample, the probe light is dispersed with a UV transmission grating onto a 256-element photodiode array for shot-to-shot detection. A chopper wheel blocks alternating pump pulses before the sample for active background subtraction. We typically average $6 \times 10^{3}$ laser pulses per delay for the $2 \mathrm{PA}$ measurements and $10^{4}$ laser pulses per delay for the TA measurements, as described below.

In the 2PA experiments, the simultaneous absorption of one pump and one probe photon occurs when the combined energy of the two-photons is resonant with a two-photon-allowed transition, but neither photon is absorbed individually. ${ }^{40-44}$ Using three different pump wavelengths $(370,390$, and $420 \mathrm{~nm})$ and a continuum probe in the range $750-400 \mathrm{~nm}$ gives overlapping spectra that cover the full range of $2 \mathrm{PA}$ transition energies from 4.6 to $6.5 \mathrm{eV}$. We scan the relative delay of the two pulses in order to account for temporal dispersion of the probe, then integrate the transient signal at each probe wavelength over a range of delay times (typically \pm 0.3 ps relative to the optimum temporal overlap) to obtain the relative $2 \mathrm{PA}$ cross-section as a function of the total pump + probe transition energy.$^{40}$ The integrated signal is independent of 
non-resonant effects, such as cross-phase modulation of the probe light. ${ }^{40,47}$ Varying the integration range confirms that there are no contributions to the signal from photoproduct transient absorption, because the integrated signal does not change when increasing the integration window outside of the region of pulse overlap. The diameters of the overlapping pump and probe beams are $\sim 300 \mu \mathrm{m}$ and $\sim 100 \mu \mathrm{m}$ at the sample, respectively, and the average energy of the pump pulse is typically $35 \mathrm{~nJ} /$ pulse. The sample for the $2 \mathrm{PA}$ measurements consists of a 1-mm path length quartz cuvette filled with a $0.5 \mathrm{M}$ solution of trans-stilbene (Aldrich, 96\%) in chloroform (Sigma-Aldrich, $\geq 99 \%$ ). We are unable to measure the 2PA spectrum of trans-stilbene in cyclohexane due to low solubility in that solvent.

Separate TA experiments probe the excited-state dynamics of trans-stilbene following one- and two-photon excitation..$^{47,48}$ One-photon excitation (1PE) experiments use pump pulses at $310 \mathrm{~nm}(4.0 \mathrm{eV})$ that are resonant with the lowest absorption band of trans-stilbene. The $\sim 0.5-\mu \mathrm{J}$ pulses are weakly focused to a diameter of $\sim 300 \mu \mathrm{m}$ at the sample, where they intersect the $\sim 100-\mu \mathrm{m}$ diameter probe beam. Non-resonant two-photon excitation (2PE) requires higherintensity pump pulses, therefore we reduce the beam diameter to $\sim 100 \mu \mathrm{m}$ at the sample and increase the pulse energy $(\sim 2 \mu \mathrm{J}$ at $475 \mathrm{~nm}$, or $\sim 3 \mu \mathrm{J}$ at $380 \mathrm{~nm})$. The intense, non-resonant pump beam induces degenerate two-photon excitation at a total transition energy equal to twice the photon energy, which is $5.2 \mathrm{eV}$ for $475-\mathrm{nm}$ irradiation and $6.5 \mathrm{eV}$ for $380-\mathrm{nm}$ irradiation. The TA measurements cover a range of delays on a quasi-logarithmic scale from -1 to $800 \mathrm{ps,} \mathrm{with}$ the relative polarization of the pump and probe set to the magic angle $\left(54.7^{\circ}\right)$ in order to exclude anisotropic effects. Samples of trans-stilbene in cyclohexane (Sigma-Aldrich, >99\%) were prepared with concentrations of $1.2 \mathrm{mM}$ for the $1 \mathrm{PE}$ measurements and $6.5 \mathrm{mM}$ for the $2 \mathrm{PE}$ measurements. The sample solution passes through a slit nozzle to form a windowless liquid stream with a path length of $300 \mu \mathrm{m}$. 


\section{RESULTS AND ANALYSIS}

One- and Two-Photon Absorption Spectroscopy

The top panel of Figure 1 shows the one-photon absorption (1PA) spectrum of transstilbene in solution. The spectrum is essentially the same in both cyclohexane and chloroform, except for a weak bathochromic shift of $4 \mathrm{~nm}$. Solvent absorption below $260 \mathrm{~nm}$ prevents us from measuring the full 1PA spectrum of trans-stilbene in chloroform. The three prominent bands in the 1PA spectrum, located near 4.1,5.4, and $6.1 \mathrm{eV}$, have been discussed extensively in the literature..$^{28,33,48,49}$ The solid vertical bars in the figure represent transition energies and oscillator strengths for one-photon allowed transitions that were calculated by Molina et al ${ }^{49}$ using CASPT2. The calculated energies are in good agreement with our experimental spectrum (see Table 1).

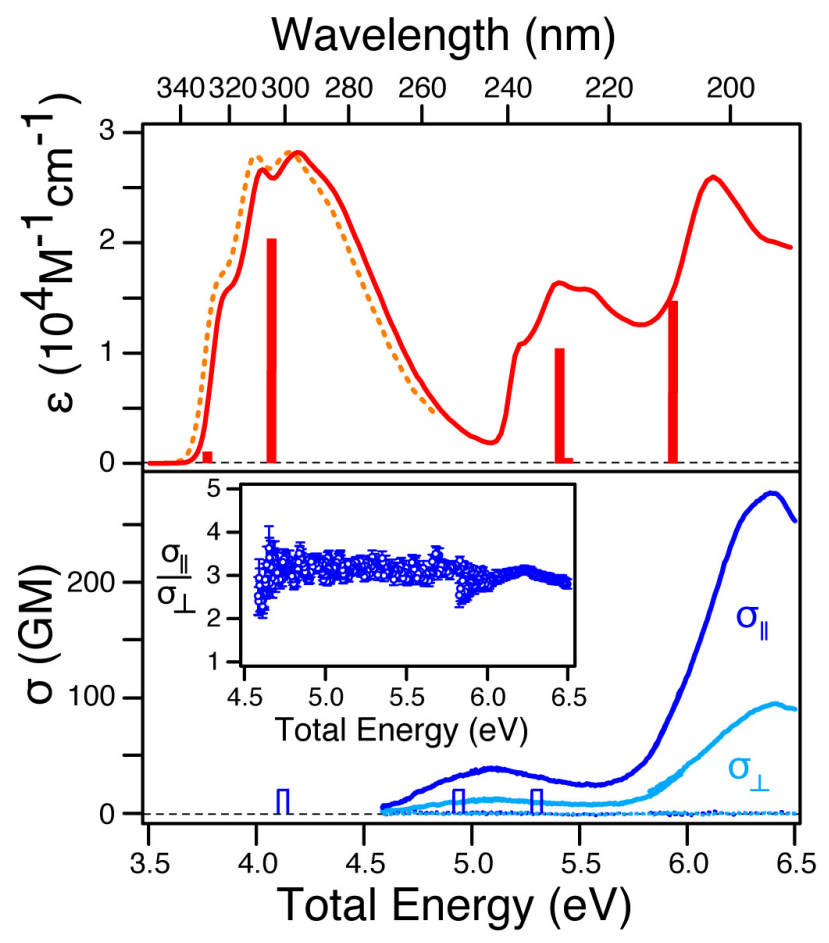

Figure 1. One- and two-photon absorption spectroscopy of trans-stilbene in solution. The top panel shows one-photon absorption spectra in cyclohexane (solid line) and in chloroform (dashed line). The bottom panel shows two-photon absorption spectra in chloroform, measured using 
both parallel (dark blue line) and perpendicular (light blue line) relative polarization of the pump and probe light. The inset shows the ratio of the two-photon cross-sections for parallel and perpendicular polarization. The solid bars in the top panel are calculated one-photon transition energies and intensities, and the hollow bars in the lower panel are two-photon transition energies, both from Ref. 49.

Table 1. One- and two-photon transition energies of trans-stilbene (in eV).

\begin{tabular}{cccc}
$\begin{array}{c}\text { Excited } \\
\text { State }\end{array}$ & Experimental $^{a}$ & $\begin{array}{c}\text { Calculated CASPT2 } \\
(\text { major configuration })^{b}\end{array}$ & $\begin{array}{c}\text { Calculated } \\
\text { SDCI/P 200 }\end{array}$ \\
\hline${ }^{1} \mathrm{~B}_{\mathrm{u}}$ & 4.03 & $3.77\left(4 \mathrm{a}_{\mathrm{u}} \rightarrow 5 \mathrm{~b}_{\mathrm{g}}\right)^{d}$ & 4.31 \\
& & $4.07\left(4 \mathrm{a}_{\mathrm{u}} \rightarrow 4 \mathrm{~b}_{\mathrm{g}}\right)^{d}$ & 4.67 \\
${ }^{1} \mathrm{~A}_{\mathrm{g}}$ & 5.12 & $4.13\left(3 \mathrm{~b}_{\mathrm{g}} \rightarrow 4 \mathrm{~b}_{\mathrm{g}}\right)$ & 4.67 \\
& & $4.95\left(4 \mathrm{a}_{\mathrm{u}}{ }^{2} \rightarrow 4 \mathrm{~b}_{\mathrm{g}}{ }^{2}\right)$ & 5.23 \\
& & $5.30\left(2 \mathrm{~b}_{\mathrm{g}} \rightarrow 4 \mathrm{~b}_{\mathrm{g}}\right)$ & 5.93 \\
${ }^{1} \mathrm{~B}_{\mathrm{u}}$ & 5.41 & $5.42\left(3 \mathrm{a}_{\mathrm{u}} \rightarrow 4 \mathrm{~b}_{\mathrm{g}}\right)$ & 5.80 \\
& & $5.42\left(4 \mathrm{a}_{\mathrm{u}} \rightarrow 5 \mathrm{~b}_{\mathrm{g}}\right)$ & 6.27 \\
& & $5.46\left(2 \mathrm{a}_{\mathrm{u}} \rightarrow 4 \mathrm{~b}_{\mathrm{g}}\right)$ & 6.60 \\
${ }^{1} \mathrm{~B}_{\mathrm{u}}$ & 6.14 & $5.95\left(3 \mathrm{a}_{\mathrm{u}} \rightarrow 5 \mathrm{~b}_{\mathrm{g}}\right)$ & 7.07 \\
${ }^{1} \mathrm{~A}_{\mathrm{g}}$ & \multirow{2}{*}{6.40} & & 6.19 \\
& & & 7.05 \\
& & & 7.15 \\
\hline
\end{tabular}

${ }^{a}$ This work. ${ }^{b}$ From Ref. $49 .{ }^{c}$ From Ref. $28 .{ }^{d}$ See Ref. 50 for a discussion about the correct ordering of the two lowest states.

The bottom panel of Figure 1 shows the two-photon absorption (2PA) spectrum of transstilbene in chloroform. We measure the broadband 2PA spectrum using both parallel and perpendicular relative polarization of the pump and probe light. In both cases, the spectrum has distinct bands centered near 5.1 and $6.4 \mathrm{eV}$, with the only difference being a cross section $(\sigma)$ that is smaller by about a factor of 3 for perpendicular polarization. The inset shows that the polarization ratio, $\sigma_{\text {para }} / \sigma_{\text {perp }}$, is constant across the entire spectrum. The figure also shows the baseline signal obtained under identical conditions for pure chloroform, and confirms that 2PA by the solvent is insignificant below $6.5 \mathrm{eV}$. The hollow vertical bars in the figure mark the 
calculated (CASPT2) transition energies for two-photon accessible states of trans-stilbene from Molina et $a l .{ }^{49}$ All of the bars have the same height in this case because those authors did not report two-photon absorption intensities.

The experimental 2PA cross-sections at the maxima of the two absorption bands are $40 \pm 16$ and $270 \pm 110 \mathrm{GM}\left(1 \mathrm{GM}=10^{-50} \mathrm{~cm}^{4} \cdot \mathrm{s} \cdot\right.$ molecule $^{-1} \cdot$ photon $\left.^{-1}\right)$, respectively, for parallel polarization of the pump and probe light. The uncertainties in the absolute 2PA cross-sections represent estimated $95 \%$ confidence intervals. As in all 2PA experiments, obtaining the absolute cross-section requires precise knowledge of the spatially dependent intensity profile, ${ }^{51}$ therefore we measure the horizontal and vertical profiles of both beams at the sample position in order to determine the spatial overlap of the two beams. Small deviations from the ideal overlap conditions and subtle imperfections of the pump and probe beams are the primary sources of uncertainty in our measurement.

Our results for the lower energy band are in excellent agreement with degenerate 2PA cross-sections in the range of 8-80 GM that were measured previously using various non-linear techniques. ${ }^{28-31,52-55}$ Although the cross-section is not necessarily the same for degenerate and non-degenerate excitation at the same total energy, we expect only small deviations in this case, because both pump and probe photons are individually non-resonant, and therefore resonanceenhancement effects are negligible.

Importantly, these are the first 2PA spectra of stilbene to be measured on a continuous energy scale, rather than point-by-point. This is also the first direct measurement of the 2PA spectrum. Previous measurements used indirect methods, such as two-photon fluorescence or thermal lensing, to measure the "action" cross-section on a point-by-point basis while tuning the excitation laser across the spectrum. ${ }^{28-34}$ Even after applying a wavelength-dependent correction to obtain the two-photon absorption from the action cross-section, ${ }^{31}$ point-by-point measurements are susceptible to variation of the pump beam conditions at each wavelength. By simultaneously measuring a broad range of the 2PA spectrum with a broadband probe pulse, our measurement is less sensitive to variations across the spectrum, and therefore provides a more accurate 
representation of the energy-dependence of the 2PA cross-section. Notably, we do not observe vibronic structure in the $2 \mathrm{PA}$ bands, as was previously reported..$^{28,32,34}$ By recording the absorption cross-section directly, our measurement is also independent of the wavelengthvarying fluorescence quantum yield..$^{28,30-34}$

The polarization dependence of the 2PA spectrum in the inset of Figure 1 confirms that only $\mathrm{A}_{\mathrm{g}}$-symmetry states contribute to the $2 \mathrm{PA}$ spectrum below $6.5 \mathrm{eV}$. The polarization ratio $\sigma_{\text {para }} / \sigma_{\text {perp }}$ depends on the relative symmetry of the two transition dipole moments associated with a transition, and therefore reveals the overall symmetry of the transition. ${ }^{56-58}$ For molecules in the $C_{2 \mathrm{~h}}$ point group, totally symmetric $\left(\mathrm{A}_{\mathrm{g}}\right)$ transitions give a ratio $\geq 4 / 3$, and non-totally symmetric $\left(\mathrm{B}_{\mathrm{g}}\right)$ transitions give a ratio $<4 / 3$. $^{56}$ The constant experimental polarization ratio of $\sim 3$ across the entire spectrum therefore indicates that only $\mathrm{A}_{\mathrm{g}}$-symmetry transitions are responsible for the $2 \mathrm{PA}$ bands below $6.5 \mathrm{eV}$. Our observation is consistent with the absence of calculated $\mathrm{B}_{\mathrm{g}}$-symmetry states within the range of our 2PA spectrum, ${ }^{28,59}$ as well as the constant polarization ratio for degenerate excitation comparing circular and linearly polarized light, $\sigma_{\text {circ }} / \sigma_{\text {para }}$, across the lowest 2PA band. ${ }^{28,30,32}$ Deviations from the planar equilibrium geometry play a negligible role in the spectrum of trans-stilbene in solution, ${ }^{30}$ therefore the polarization ratio provides a good measure of the electronic symmetries of the two-photon accessible excited states.

\section{Excited-State Dynamics Following One- and Two-Photon Excitation}

Figure $2 \mathrm{~A}$ shows the evolution of the transient absorption spectrum of trans-stilbene following excitation with one-photon at $310 \mathrm{~nm}$ (total energy of $4.0 \mathrm{eV}$ ). Consistent with earlier studies, ${ }^{8,12,15,18,60-62}$ the TA spectrum is dominated by an excited-state absorption (ESA) band centered near $585 \mathrm{~nm}$ and a weaker stimulated emission (SE) band near $365 \mathrm{~nm}$. The asymmetry of the ESA band, including a weak shoulder near $550 \mathrm{~nm}$, was previously assigned as a vibronic progression in the $S_{n} \leftarrow S_{1}$ transition. ${ }^{12,63-67}$ Both the positive ESA band at $585 \mathrm{~nm}$ and the negative SE band at $365 \mathrm{~nm}$ decay to the baseline on a timescale that is consistent with the previously reported lifetime of $69 \pm 11$ ps for trans-stilbene in cyclohexane. ${ }^{10}$ We also observe very weak 
shifting and narrowing of the spectrum within the first $10-20$ ps due to vibrational cooling in the excited state. ${ }^{12}$

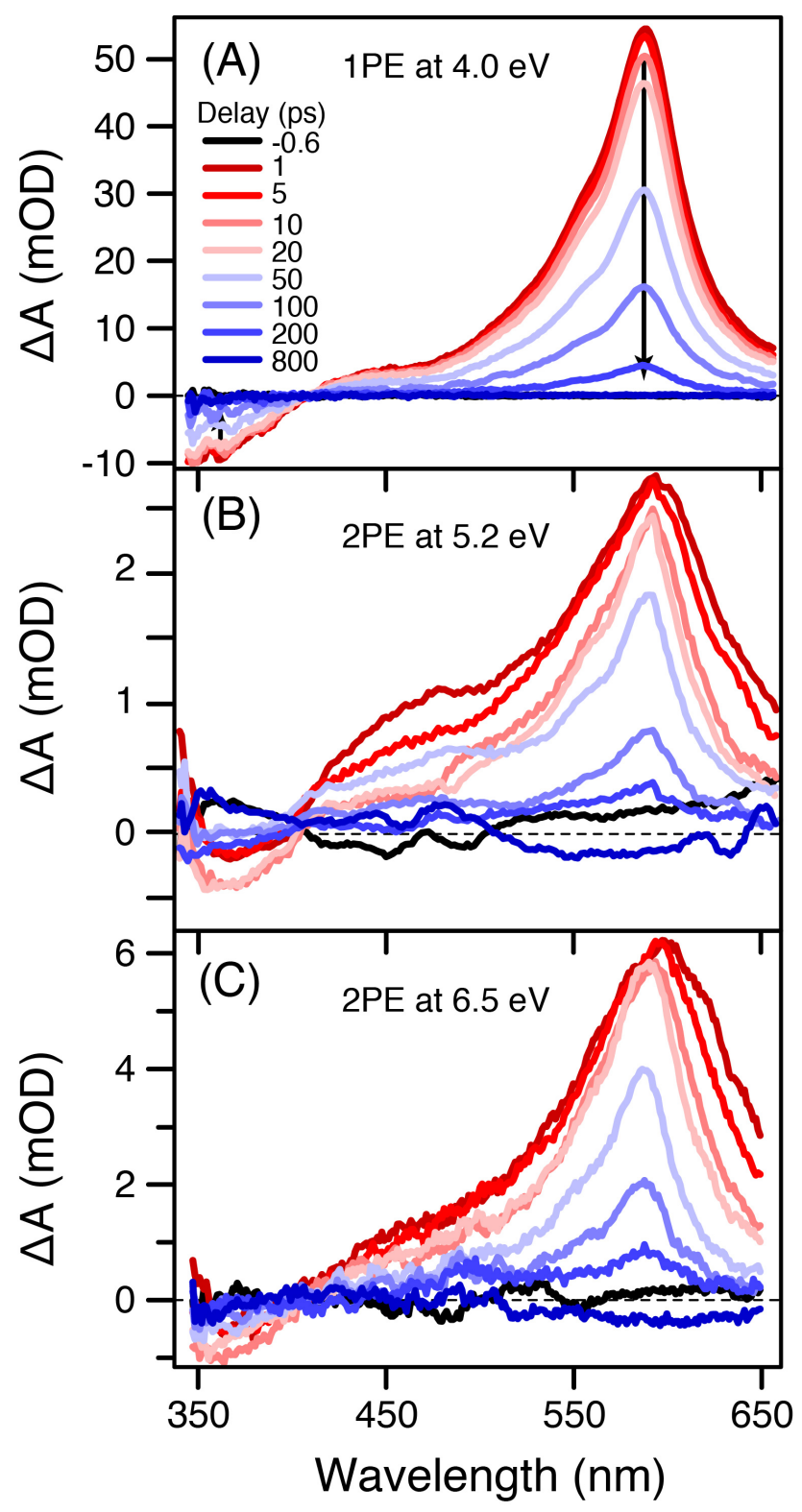

Figure 2. Transient absorption spectra of trans-stilbene following one-photon excitation at 310 $\mathrm{nm}(\mathrm{A})$, two-photon excitation at $475 \mathrm{~nm}(\mathrm{~B})$, and two-photon excitation at $380 \mathrm{~nm}(\mathrm{C})$. The spectra in (B) are averaged over three adjacent time points in order to improve the signal-tonoise ratio. 
Based on previous interpretations of the excited-state dynamics of transstilbene, $8,14,15,18,60,61$ we model the ps-scale relaxation following one-photon excitation with a sequential, two-step kinetic scheme.

$$
S_{1}^{*} \stackrel{k_{1}}{\rightarrow} S_{1} \stackrel{k_{2}}{\rightarrow} S_{0}
$$

The first species represents vibrationally excited stilbene in the first excited electronic state, $\mathrm{S}_{1}{ }^{*}$, which relaxes to the thermally populated $S_{1}$ state before returning to the ground electronic state. The TA signal is insensitive to the branching between cis and trans isomers when returning to the ground state, because neither of the ground-state species absorb in the probe window.

A global fit to the broadband TA data using this simple kinetic model gives time constants of $\left(k_{1}\right)^{-1}=8 \pm 2$ ps and $\left(k_{2}\right)^{-1}=78 \pm 6$ ps (95\% confidence) for the two steps in the sequential relaxation. Although the excitation at $310 \mathrm{~nm}$ deposits very little excess energy above the $S_{1}$ band origin, ${ }^{68}$ we include the $S_{1}{ }^{*}$ state in the model in order to obtain high-quality fits to the data and for consistency with the analysis at higher excitation energies. Species-associated spectra (SAS) obtained from the best fit to the transient absorption data for one-photon excitation at $4.0 \mathrm{eV}$ are shown in the top panel of Figure 3. The slight narrowing and shifting of the ESA band between the first and second species is characteristic of vibrational cooling in the excited state. ${ }^{12,14,18}$ 


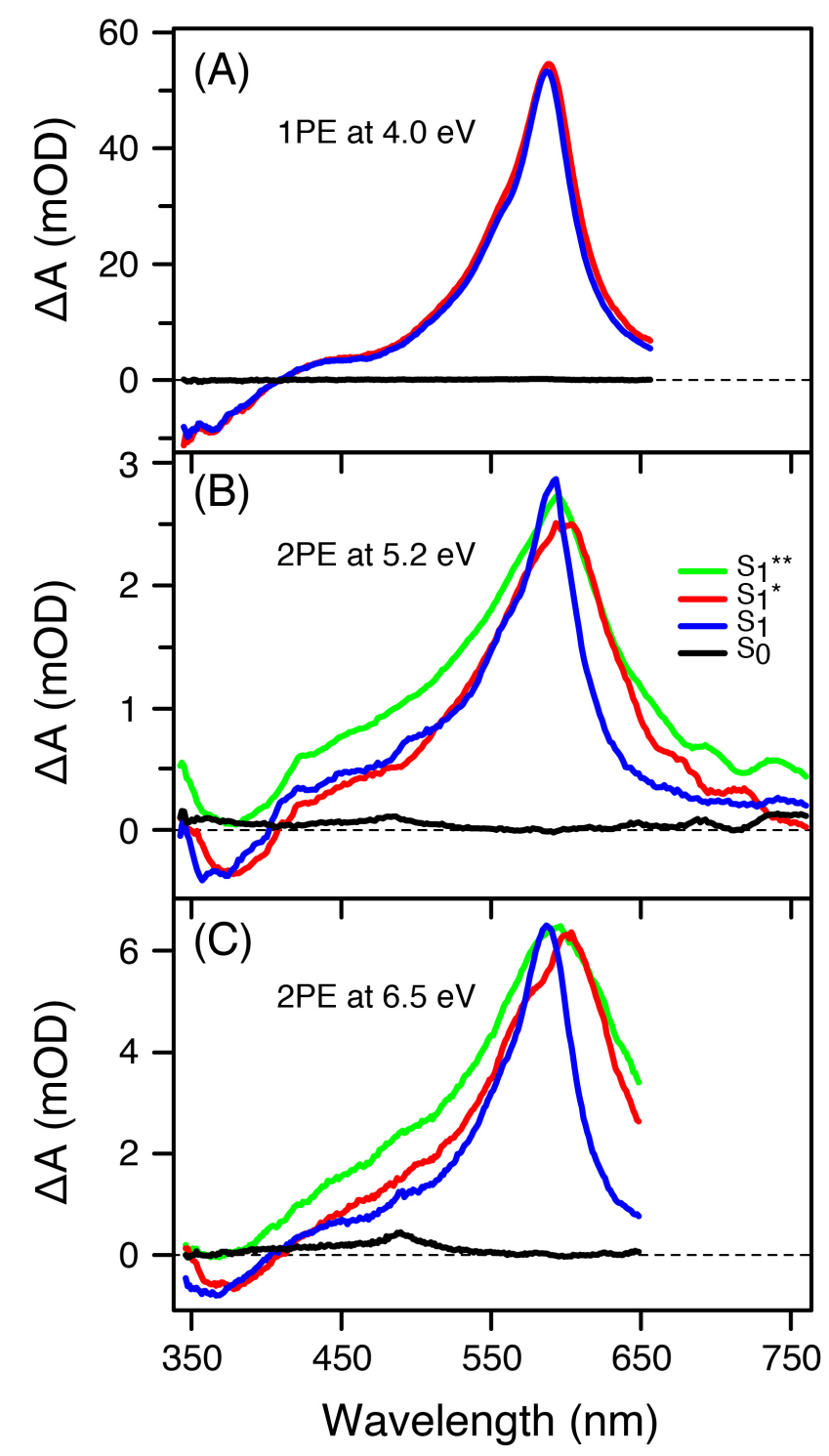

Figure 3. Species-associated spectra (SAS) from global fits to the transient absorption data using the sequential kinetics models described in the text. The spectra represent species from onephoton excitation at $310 \mathrm{~nm}(\mathrm{~A})$, two-photon excitation at $475 \mathrm{~nm}(\mathrm{~B})$, and two-photon excitation at $380 \mathrm{~nm}(\mathrm{C})$.

The lower two panels of Figure 2 show the TA spectrum following two-photon excitation at $475 \mathrm{~nm}$ (total energy $5.2 \mathrm{eV}$ ) and $380 \mathrm{~nm}$ (total energy $6.5 \mathrm{eV}$ ), respectively. The TA signals are much weaker than for 1PE due to a substantially smaller cross-section for two-photon 
excitation, that is only partially offset by the higher concentration and more intense pump pulses. Additionally, the two-photon process depends quadratically on the excitation intensity, which amplifies small fluctuations of the pump pulse intensity and further increases the noise level in the 2PE experiments. As expected from the relative 2PA cross-sections, exciting the lowerenergy band at $5.2 \mathrm{eV}$ with two photons produces a weaker TA signal than does exciting the higher-energy band at $6.5 \mathrm{eV}$. To compensate for the lower signal-to-noise ratio in the lowerenergy $2 \mathrm{PE}$ scan, we average three adjacent time points to obtain the TA spectra in Figure $2 \mathrm{~B}$. Figure S1 in the Supporting Information compares the ps-scale time evolution of the ESA and SE bands for all three excitation pathways.

Despite the lower signal-to-noise ratio for the two-photon excitation experiments, we clearly observe additional spectral evolution on a timescale of 1-2 ps that is not observed following one-photon excitation into the lowest-energy absorption band. In order to model the additional spectral evolution, we add an extra species to the sequential kinetic model to represent a precursor to $S_{1}{ }^{*}$.

$$
S_{1}^{* *} \stackrel{k_{1}}{\rightarrow} S_{1}^{*} \stackrel{k_{2}}{\rightarrow} S_{1} \stackrel{k_{3}}{\rightarrow} S_{0}
$$

The lower two panels of Figure 3 show the SAS obtained from global fits to the ps-scale transient absorption data using this four-state model. The additional step in the relaxation reveals spectral narrowing (primarily on the high-energy side of the ESA band) with time constants of $1.9 \pm 1.2 \mathrm{ps}$ and $1.5 \pm 0.3 \mathrm{ps}$ following $2 \mathrm{PE}$ at 5.2 and $6.5 \mathrm{eV}$, respectively. In both cases, the initial relaxation is followed by two slower steps that occur on the same timescales as the relaxation following $1 \mathrm{PE}$.

The similar spectra of all three species in the $2 \mathrm{PE}$ experiments (i.e., $\mathrm{S}_{1}{ }^{* *}, \mathrm{~S}_{1}{ }^{*}$, and $\mathrm{S}_{1}$ ) suggest that $S_{1}^{* *}$ represents another vibrationally excited state of $S_{1}$. Therefore, we propose that $\mathrm{S}_{1}{ }^{* *}$ represents a highly non-statistical distribution of vibrational energy in $S_{1}$ following rapid (sub-ps) internal conversion from the initially excited state, $\mathrm{S}_{\mathrm{n}}$. Intramolecular vibrational redistribution (IVR) produces a more statistical distribution of vibrational energy, represented by $\mathrm{S}_{1}{ }^{*}$, within the next 1-2 ps. The red-shift and broadening of the $\mathrm{S}_{1}{ }^{*}$ spectrum is significantly more 
pronounced for excitation to the higher-lying states than it is following $1 \mathrm{PE}$ at $310 \mathrm{~nm}$, because of the larger amount of excess energy deposited into the molecule. The $\sim 8$ ps relaxation time of $\mathrm{S}_{1}{ }^{*}$ is consistent with vibrational cooling (VC) of the hot excited state.,8,,60,61,68 Both the vibrational cooling time and the $\sim 80$ ps excited-state (ES) lifetime of $S_{1}$ are consistent with the $1 \mathrm{PE}$ result. Based on this picture, we re-label the time constants from the two kinetic models to reflect the underlying process in each step (i.e., $\tau_{\mathrm{IVR}}, \tau_{\mathrm{VC}}$, and $\tau_{\mathrm{ES}}$ ). Table 2 summarizes the time constants for all three excitation energies.

Table 2. Time constants for intramolecular vibrational relaxation $\left(\tau_{\mathrm{IVR}}\right)$, vibrational cooling $\left(\tau_{\mathrm{VC}}\right)$, and relaxation of the excited state $\left(\tau_{\mathrm{ES}}\right)$ of trans-stilbene from fits to the transient absorption spectra using sequential kinetic models.

\begin{tabular}{lccc}
\hline & $\tau_{\text {IVR }} / \mathrm{ps}$ & $\tau_{\mathrm{VC}} / \mathrm{ps}$ & $\tau_{\mathrm{ES}} / \mathrm{ps}$ \\
\hline $1 \mathrm{PE}(4.0 \mathrm{eV})$ & - & $7.6(1.8)$ & $78(6)$ \\
$2 \mathrm{PE}(5.2 \mathrm{eV})$ & $1.9(1.2)$ & $6.2(2.5)$ & $83(17)$ \\
$2 \mathrm{PE}(6.5 \mathrm{eV})$ & $1.5(0.3)$ & $9.3(1.5)$ & $82(5)$ \\
\hline
\end{tabular}

We see evidence of the faster $S_{n} \rightarrow S_{1}$ electronic relaxation on a sub-ps timescale, as shown in Figure 4. The figure compares the evolution of the transient absorption spectra within the first $600 \mathrm{fs}$ following one- and two-photon excitation of trans-stilbene. The most obvious difference between the two transient spectra is a new, short-lived absorption band in the higher-energy region of the spectrum following $2 \mathrm{PE}$. The short-lived absorption band precedes the formation of the 585-nm ESA band following two-photon excitation (Figure 4B), whereas the rise of the 585nm ESA band is instrument-limited following one-photon excitation (Figure 4A). A delayed onset of the $S_{1}$ excited-state absorption and stimulated emission bands was also observed by Kovalenko et al. ${ }^{12}$ following one-photon excitation into the second absorption band at $226 \mathrm{~nm}$, therefore we interpret the delayed appearance of the 585-nm ESA band as relaxation from the initially excited electronic state $S_{n}$ to the vibrationally hot $S_{1}^{* *}$. 


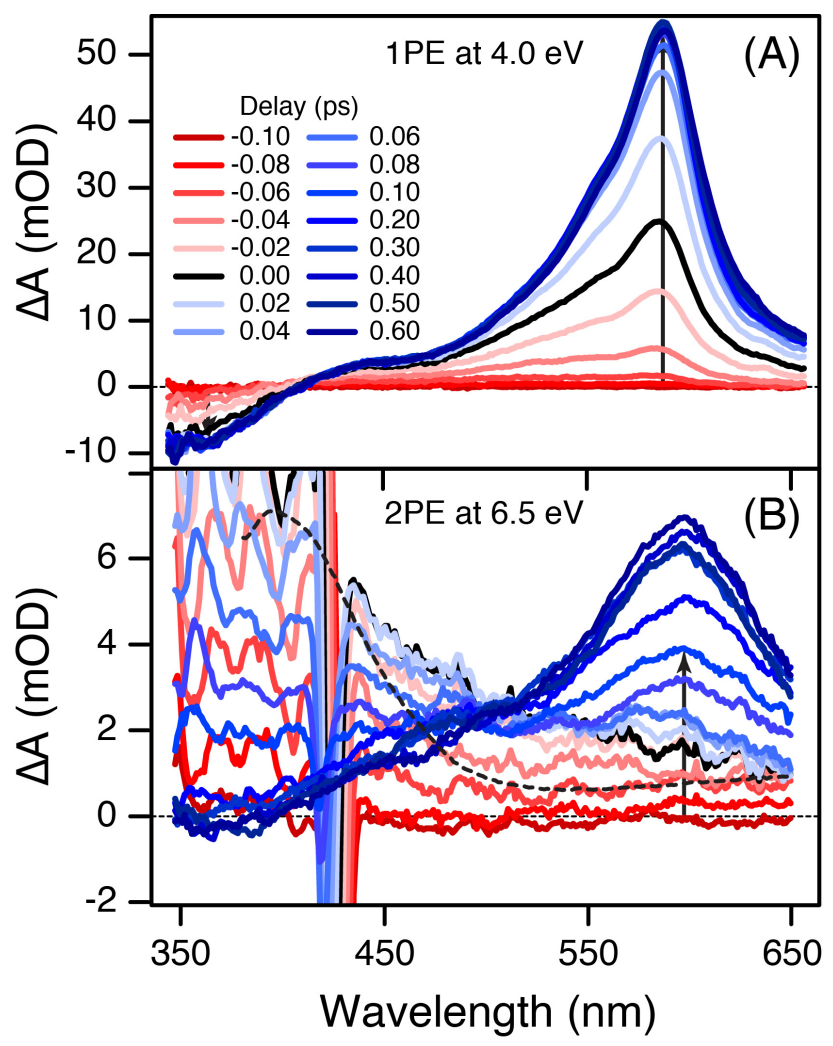

Figure 4. Transient absorption spectra of trans-stilbene at early time delays following onephoton excitation at $310 \mathrm{~nm}$ (A) and two-photon excitation at $380 \mathrm{~nm}$ (B). The black arrows show the rising excited-state absorption signal up to a delay of $100 \mathrm{fs}$. Note that the rise of the ESA band is complete with the instrument response time of $\sim 75$ fs in (A), but the signal continues to rise for several hundred fs in (B). The thin dashed line in (B) is the scaled twophoton absorption spectrum, and therefore represents an upper limit for the 2PA contribution to the transient absorption signal at 0 ps delay (black line).

Figure 5 shows the temporal evolution of the sub-ps transient absorption signals at several probe wavelengths. Following two-photon excitation, the short-lived TA signal at 400 $\mathrm{nm}$ decays within $\sim 100 \mathrm{fs}$, with a weak tail extending to a few hundred fs. The fleeting transient absorption signal is at least partially due to ESA from an electronic state above $\mathrm{S}_{1}$, as discussed below. The initial decay of this band is comparable to the instrument response time of $\sim 75$ fs (FWHM), reflecting a very short lifetime for the higher-lying electronically excited state. The 
slower decaying tail at $400 \mathrm{~nm}$, which matches the rise at $600 \mathrm{~nm}$, could be either an intermediate electronic state, or a very non-statistical distribution of vibrational energy in $\mathrm{S}_{1}$ that precedes even $\mathrm{S}_{1}^{* *}$.

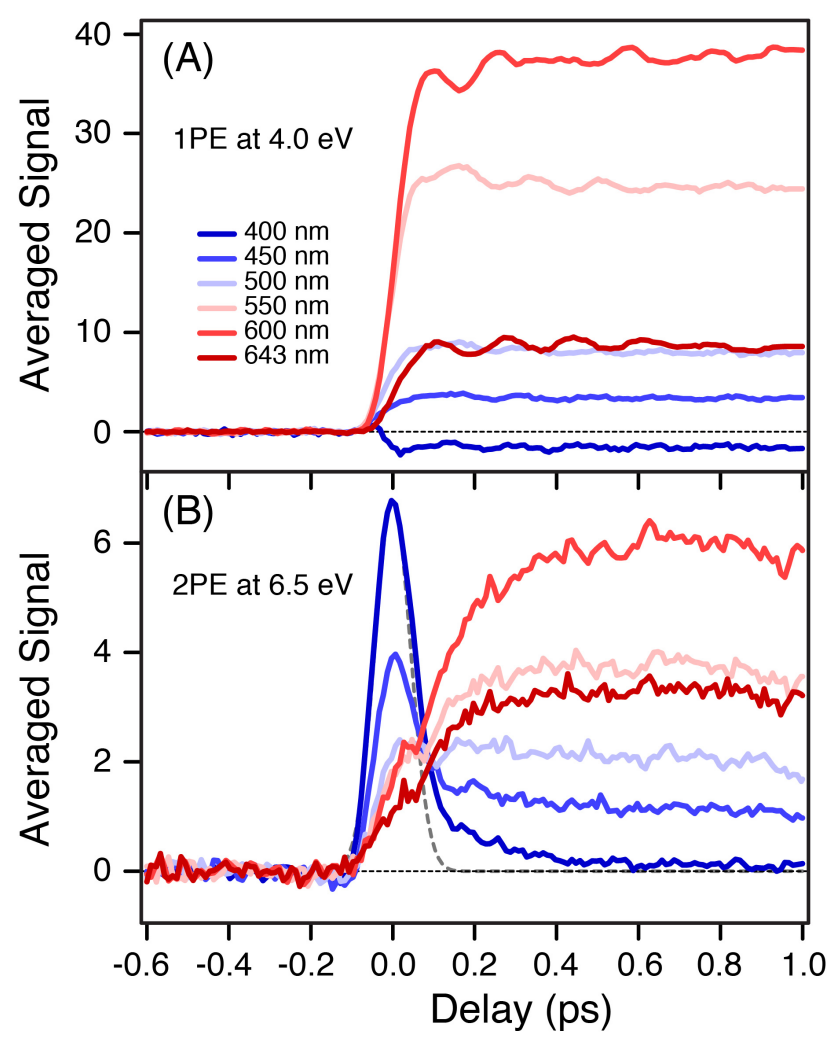

Figure 5. Evolution of the transient absorption of trans-stilbene at early time delays following one-photon excitation at $310 \mathrm{~nm}(\mathrm{~A})$ and two-photon excitation at $380 \mathrm{~nm}(\mathrm{~B})$. Each transient is averaged over a $\sim 13 \mathrm{~nm}$ range of probe wavelengths (11 adjacent pixels) to improve the signalto-noise ratio. The dashed line in $(\mathrm{B})$ is the instrument response function for the two-photon excitation experiment, based on the coherent Raman response of the pure solvent at $425 \mathrm{~nm}$.

Non-resonant two-photon (pump + probe) absorption could also contribute to the shortlived TA signal within the instrument response time. However, the spectral shape of the TA feature in Figure 4B is inconsistent with the broadband 2PA spectrum in Figure 1. Most notably, the short-lived TA band decreases monotonically across the spectrum, with no evidence of the 
2PA minimum at $5.6 \mathrm{eV}$. The $2 \mathrm{PA}$ minimum would be observed at a probe wavelength of 530 $\mathrm{nm}$ in the case of the 380-nm pump (i.e., $3.3 \mathrm{eV}$ pump $+2.3 \mathrm{eV}$ probe), as shown by the dashed line in Figure 4B. The 2PA signal cannot exceed the total TA signal at any single wavelength, therefore the dashed line represents the upper limit of the signal due to non-resonant two-photon (pump + probe) absorption, and indicates that the ESA of an electronically excited state is responsible for at least a majority of the time-zero TA signal in the region $450-600 \mathrm{~nm} .^{69}$ The isosbestic point near $500 \mathrm{~nm}$ further supports the picture of a sequential population decay from $\mathrm{S}_{\mathrm{n}}$ to $\mathrm{S}_{1}^{* *}$ following $2 \mathrm{PE}$ at $6.5 \mathrm{eV}$.

The situation is less clear following $2 \mathrm{PE}$ at $5.2 \mathrm{eV}$, due to the lower signal-to-noise ratio, and because the Raman response of the solvent induced by 475-nm pump light overlaps a broader region of the transient absorption spectrum. However, subtraction of the solvent signal (see the Supporting Information) indicates that there is probably a similar short-lived ESA band in that case, as well. Therefore, the sub-ps evolution of the TA spectrum following $2 \mathrm{PE}$ reveals internal conversion from a higher-lying electronic state (or states) and the formation of the highly vibrationally excited $\mathrm{S}_{1}{ }^{* *}$ at both excitation energies.

\section{DISCUSSION}

One- and two-photon absorption spectroscopy reveals a great deal of information about the electronic structure of trans-stilbene. The one- and two-photon spectra are complementary, because different transitions are allowed in the two cases based on parity selection rules and the $C_{2 \mathrm{~h}}$ symmetry of the planar molecule. The one-photon spectrum of trans-stilbene has been

discussed widely, ${ }^{28,33,48,49}$ therefore we describe primarily the 2PA spectrum. We observe two distinct, broad transitions near 5.1 and $6.4 \mathrm{eV}$, respectively. These two bands represent the two excited electronic states that we access in the transient absorption experiments.

As a first step toward identifying the two-photon-accessible excited states, the 2PA polarization ratio (Figure 1, inset) indicates that only $\mathrm{A}_{\mathrm{g}}$-symmetry states contribute to the twophoton spectrum below $6.5 \mathrm{eV}$. Calculations show that there are several two-photon accessible 
states with $\mathrm{A}_{\mathrm{g}}$ symmetry in the region of our experiment. ${ }^{20,21,28,49,59,70-72}$ Although we compare our experimental transition energies with the CASPT2 calculations by Molina et al., ${ }^{49}$ those authors did not report 2PA intensities, therefore we look to lower-level configuration interaction calculations to help identify which states contribute to the 2PA spectrum (see Table 1). The latter calculations suggest that the lowest-lying $\mathrm{A}_{\mathrm{g}}$-symmetry state has a much smaller 2PA crosssection than higher-lying states of the same symmetry. ${ }^{28,73}$ The calculations are consistent with earlier observations of a very weak $2 \mathrm{PA}$ band near $4.1 \mathrm{eV}$ that lies below the range of our 2PA spectrum. $^{28,34}$ In contrast, the second-lowest state with $\mathrm{A}_{\mathrm{g}}$-symmetry has a larger two-photon absorption cross-section. ${ }^{28}$ This transition has significant doubly excited character $\left(4 \mathrm{a}_{\mathrm{u}}{ }^{2} \rightarrow 4 \mathrm{~b}_{\mathrm{g}}{ }^{2}\right),{ }^{49}$ and was previously attributed to the $2 \mathrm{PA}$ band near $5.1 \mathrm{eV}$. However, the identity of the higherenergy 2PA band near $6.4 \mathrm{eV}$ is less clear. The calculations do not reveal a single electronic state that is responsible for this more intense, higher-lying two-photon transition, but rather several states that may contribute (see Table 1$).{ }^{28}$ Given the high density of electronic states and significant configurational mixing, we expect strong coupling among these higher-lying electronic states.

The state diagram in Figure 6 provides a reference point for describing the excited-state dynamics in the context of the one- and two-photon absorption spectroscopy of trans-stilbene. The schematic energy-level diagram in the figure also incorporates information from electronic structure calculations, ${ }^{24,26,74}$ as well as previous dynamics measurements. ${ }^{8-12,14-18,60-62}$ The dynamics following excitation into the first absorption band have been discussed widely; however, the dynamics following excitation to the higher-lying states have received much less attention. $^{12,16,17,39}$ 


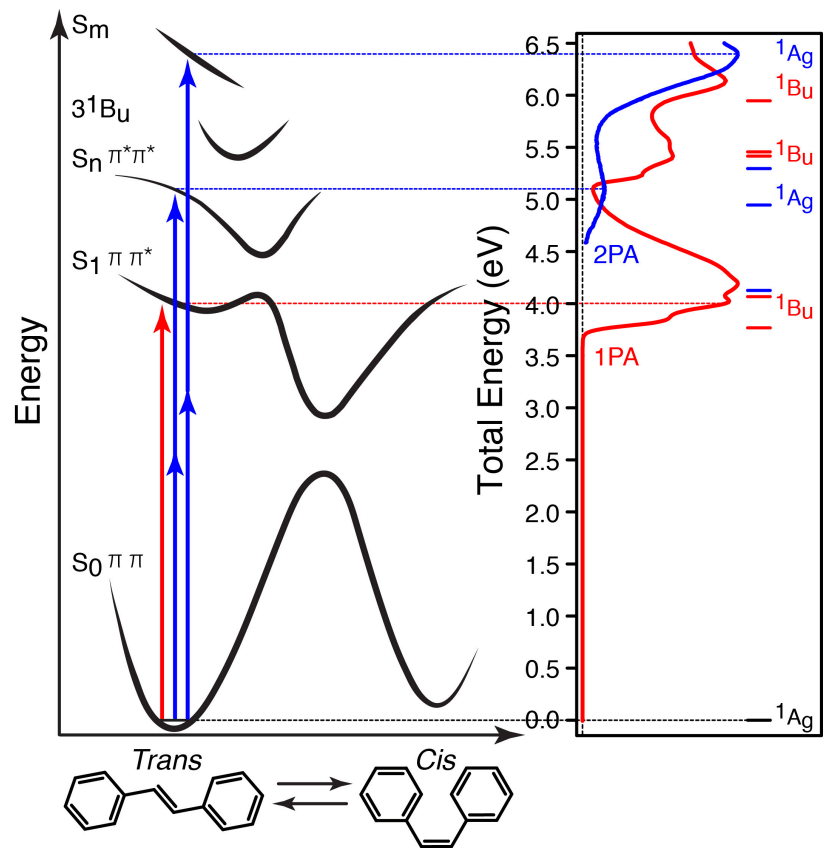

Figure 6. Schematic diagram comparing potential energies curves for several excited states of trans-stilbene with the one- and two-photon absorption spectra from the current work and with calculated transition energies from Ref. 49.

Two-photon excitation at $5.2 \mathrm{eV}$ accesses a state with significant doubly excited $\left(\pi^{*} \pi^{*}\right)$ character, and therefore correlates diabatically to the ground-state of the cis isomer. Despite an electronic configuration that is diabatically similar to the ground state of the product, the ps-scale transient absorption spectroscopy and excited-state lifetimes following $2 \mathrm{PE}$ of this state are identical to the ps-scale results following 1PE of the lowest $\left(\pi^{*} \pi\right)$ excited state at $4.0 \mathrm{eV}$. Similar ps-scale dynamics are also observed following two-photon excitation to a higher-lying state at 6.5 $\mathrm{eV}$. The similar result for all three excitation energies indicates that the isomerization reaction follows an analogous path on the adiabatic $S_{1}$ state, regardless of the initial excitation level. In other words, rapid electronic relaxation from the higher-lying excited state puts the molecule on $S_{1}$ before crossing a barrier along the torsional coordinate that inhibits motion to the $\mathrm{S}_{1}-\mathrm{S}_{0}$ conical intersection. That is to say, the molecule probably does not reach a perpendicular geometry in the higher-excited state before relaxing onto $S_{1}$. 
Fast electronic relaxation from the higher-lying states to the lowest excited state is predicted by Kasha's rule. Surprisingly, however, the excess vibrational energy following internal conversion to $S_{1}$ does not efficiently couple into the reaction coordinate. There are two explanations for this behavior. Either IVR is incomplete, and therefore the excess energy does not accelerate the barrier crossing before dissipating to the solvent, or else IVR is complete, but there is simply too little energy overall to accelerate the reaction substantially. Assuming equipartition, Two-photon excitation at $5.2 \mathrm{eV}$ deposits only $\sim 31 \mathrm{~cm}^{-1}$ of excess energy per mode, and $2 \mathrm{PE}$ at $6.5 \mathrm{eV}$ deposits $\sim 177 \mathrm{~cm}^{-1}$. Given the observation of rapid IVR in the first 1-2 ps following internal conversion to the $S_{1}$ state, the second situation seems more likely.

The 1-2 ps IVR process that we observe following two-photon excitation indicates an added degree of structural relaxation compared with one-photon excitation to $S_{1}$ directly. The highly non-statistical distribution of vibrational energy in $S_{1}^{* *}$ is a result of the internal conversion from a higher-lying electronic state. Although, that excess vibrational energy does not couple efficiently into the torsional motion of the molecule, the center wavelength of the ESA band temporarily shifts to lower energy in going from $S_{1}{ }^{* *}$ to $S_{1}{ }^{*}$, and then shifts back upon cooling to the $S_{1}$ equilibrium state (see the SAS in Figure 3). This behavior provides a sensitive probe of the energy disposal following internal conversion by probing the transient population of one or a few specific vibrational modes of the molecule that are responsible for changing the Franck-Condon overlap with the higher-lying state in the transition. Similar effects are responsible for coherent oscillations of the 585-nm ESA band within the first ps following 1PE (see Figure 5), which reveals an in-plane $\mathrm{C}=\mathrm{C}-\mathrm{Ph}$ bending motion on the $\mathrm{S}_{1}$ excited state. ${ }^{75}$ Bao and Weber observed coherent oscillations in a different mode that they assigned as torsional motion of the phenyl groups following one-photon excitation at $5.93 \mathrm{eV}$ in the gas phase. ${ }^{16,17}$ In contrast, we do not observe any coherent oscillations following $2 \mathrm{PE}$ at $5.2 \mathrm{or} 6.5 \mathrm{eV}$. Either the very short electronic lifetimes lead to rapid dephasing, or else electronic spectroscopy is simply not sensitive to the specific motions that are induced by excitation to these particular excited states. Electronic spectroscopy is a relatively insensitive measure of the vibrational dynamics, 
therefore transient Raman spectroscopy ${ }^{62,68,76-82}$ could provide additional insight following excitation to the higher-lying states of trans-stilbene.

The transient absorption spectroscopy measurements also reveal a very short-lived excited state species following excitation to the higher-lying states. Unfortunately, we are not able to clearly resolve the ESA spectra in order to distinguish different states that may be involved in the relaxation pathways following 2PE. Nevertheless, the TA spectra provide a direct probe of the timescales for electronic relaxation from the higher-lying states to $S_{1}$. Consistent with Kasha's rule, internal conversion to $S_{1}$ occurs very rapidly, even though the molecule starts in a state with different electronic symmetry. One possible pathway to explain the similar shortlived ESA spectra for the two excitation energies is that the molecule relaxes from the initially excited state to the lowest-lying $\mathrm{A}_{\mathrm{g}}$-symmetry state in $<75 \mathrm{fs}$, and then relaxes to $\mathrm{S}_{1}{ }^{* *}$ on the $\sim 100$ fs timescale, in which case the ESA contribution that we observe in Figure 4B probes the population of the lowest-energy $\mathrm{A}_{\mathrm{g}}$ state.

\section{CONCLUSIONS}

Complementary two-photon absorption spectroscopy and two-photon excitation measurements give new insight into the dynamics of the higher-lying excited states of transstilbene in solution. Spectroscopy is an essential tool for determining the excited-state energies and identifying the initial excited states that are populated by one- and two-photon transitions. For example, the absorption of a single photon at $310 \mathrm{~nm}$ excites the molecule slightly above the origin of the lowest excited state, depositing a small amount of excess vibrational energy that dissipates to the solvent in $\sim 8 \mathrm{ps}^{68}$ The molecule then returns to the ground electronic state in $\sim 80$ ps by passing through a conical intersection with roughly 1:1 branching between the cis and trans isomers. ${ }^{3}$ Two additional relaxation processes are observed within the first few ps following excitation with two-photons to higher-lying excited-states. Internal conversion from the initially excited state, $S_{n}$, to a non-equilibrium $S_{1}{ }^{* *}$ state occurs within $\sim 100 \mathrm{fs}$, followed by 
rapid IVR (1-2 ps). The ensuing dynamics are exactly the same as the case of one-photon excitation.

Higher-lying excited states are a frontier in the field of chemical dynamics, because those states present significant challenges for both experiment and theory. We hope that our 2PA and TA measurements will stimulated renewed computational interest in the higher-lying states of trans-stilbene and other model systems by providing new spectroscopic benchmarks for high level calculations of the transition energies and intensities of two-photon allowed states, as well as experimental evidence of the electronic and nuclear relaxation dynamics. Additional experimental studies with better time resolution or more sensitive probes of the vibrational dynamics will provide further insight into the structural relaxation and isomerization dynamics of trans-stilbene following excitation to the higher-lying excited states.

\section{ASSOCIATED CONTENT}

\section{Supporting Information}

Additional figures include kinetic traces showing the evolution of the ESA and SE bands for all three excitation energies, decay-associated spectra from global fits to the data using a sum of exponentials, the time-dependent populations of the SAS in Figure 3, the sub-ps evolution of the TA spectrum following $2 \mathrm{PE}$ at $475 \mathrm{~nm}$, and power dependence measurements for the $2 \mathrm{PE}$ experiments. This material is available free of charge via the Internet at http://pubs.acs.org.

\section{AUTHOR INFORMATION}

\section{Corresponding Author}

"E-mail: elles@ku.edu

\section{Present Addresses}

${ }^{\dagger}$ Spectra Group Limited, Inc., Millbury, Ohio 43447

Department of Polymer Science, University of Akron, Akron, Ohio 44325 


\section{ACKNOWLEDGEMENTS}

The work in this publication was supported by the National Science Foundation (NSF) through a CAREER Award (CHE-1151555). TAT acknowledges support through the NSF Research Experience for Undergraduates program at the University of Kansas (CHE-1004897).

\section{REFERENCES}

(1) Thomas, J. K. Higher Excited States in Multiphoton Photochemical Reactions "Hint" Toward Rapid Chemistry. J. Phys. Chem. Lett. 2014, 5, 2586-2587.

(2) Kasha, M. Characterization of Electronic Transitions in Complex Molecules. Discuss. Faraday Soc. 1950, 9, 14-19.

(3) Waldeck, D. H. Photoisomerization Dynamics of Stilbenes. Chem. Rev. 1991, 91, 415436.

(4) Meier, H. The Photochemistry of Stilbenoid Compounds and Their Role in Materials Technology. Angew. Chem. Int. Ed. Engl. 1992, 31, 1399-1420.

(5) Irie, M. Diarylethenes for Memories and Switches. Chem. Rev. 2000, 100, 1685-1716.

(6) Kay, E. R.; Leigh, D. A.; Zerbetto, F. Synthetic Molecular Motors and Mechanical Machines. Angew. Chem. Int. Ed. 2007, 46, 72-191.

(7) Budyka, M. F. Diarylethylene Photoisomerization and Photocyclization Mechanisms. Russ. Chem. Rev. 2012, 81, 477-493.

(8) Hochstrasser, R. M. Picosecond Processes in the Isomerism of Stilbenes. Pure Appl. Chem. 1980, 52, 2683-2691.

(9) Sension, R. J.; Repinec, S. T.; Hochstrasser, R. M. Femtosecond Laser Study of Energy Disposal in the Solution Phase Isomerization of Stilbene. J. Chem. Phys. 1990, 93, 9185-9188.

(10) Rice, J. K.; Baronavski, A. P. Ultrafast Studies of Solvent Effects in the Isomerization of cis-Stilbene. J. Phys. Chem. 1992, 96, 3359-3366. 
(11) Baskin, J. S.; Bañares, L.; Pedersen, S.; Zewail, A. H. Femtosecond Real-Time Probing of Reactions. 20. Dynamics of Twisting, Alignment, and IVR in the trans-Stilbene Isomerization Reaction. J. Phys. Chem. 1996, 100, 11920-11933.

(12) Kovalenko, S. A.; Schanz, R.; Hennig, H.; Ernsting, N. P. Cooling Dynamics of an Optically Excited Molecular Probe in Solution from Femtosecond Broadband Transient Absorption Spectroscopy. J. Chem. Phys. 2001, 115, 3256-3273.

(13) Fuß, W.; Kosmidis, C.; Schmid, W. E.; Trushin, S. A. The Photochemical cis-trans Isomerization of Free Stilbene Molecules Follows a Hula-Twist Pathway. Angew. Chem. Int. Ed. 2004, 43, 4178-4182.

(14) Weigel, A.; Ernsting, N. P. Excited Stilbene: Intramolecular Vibrational Redistribution and Solvation Studied by Femtosecond Stimulated Raman Spectroscopy. J. Phys. Chem. B 2010, $114,7879-7893$.

(15) Kovalenko, S. A.; Dobryakov, A. L.; Ioffe, I.; Ernsting, N. P. Evidence for the Phantom State in Photoinduced cis-trans isomerization of Stilbene. Chem. Phys. Lett. 2010, 493, 255-258. (16) Bao, J.; Weber, P. M. Ultrafast Dynamics of Highly Excited trans-Stilbene: A Different Twist. J. Phys. Chem. Lett. 2010, 1, 224-227.

(17) Bao, J.; Weber, P. M. Electronic Effects on Photochemistry: The Diverse Reaction Dynamics of Highly Excited Stilbenes and Azobenzene. J. Am. Chem. Soc. 2011, 133, 41644167.

(18) Kovalenko, S. A.; Dobryakov, A. L. On the Excitation Wavelength Dependence and Arrhenius Behavior of Stilbene Isomerization Rates in Solution. Chem. Phys. Lett. 2013, 570, 56-60.

(19) Orlandi, G.; Siebrand, W. Model for Direct Photoisomerization of Stilbene. Chem. Phys. Lett. 1975, 30, 352-354.

(20) Orlandi, G.; Palmieri, P.; Poggi, G. An ab Initio Study of the Cis-Trans Photoisomerization of Stilbene. J. Am. Chem. Soc. 1979, 101, 3492-3497. 
Cooperating Rings in cis-Stilbene Lead to an $\mathrm{S}_{0} / \mathrm{S}_{1}$ Conical Intersection. J. Phys. Chem. A 1997, 101, 3841-3847.

(22) Orlandi, G.; Gagliardi, L.; Melandri, S.; Caminati, W. Torsional Potential Energy

Surfaces and Vibrational Levels in trans Stilbene. J. Mol. Struct. 2002, 612, 383-391.

(23) Kwasniewski, S. P.; Claes, L.; François, J.-P.; Deleuze, M. S. High Level Theoretical

Study of the Structure and Rotational Barriers of trans-Stilbene. J. Chem. Phys. 2003, 118, 78237836.

(24) Quenneville, J.; Martinez, T. J. Ab Initio Study of Cis-Trans Photoisomerization in Stilbene and Ethylene. J. Phys. Chem. A 2003, 107, 829-837.

(25) Improta, R.; Santoro, F. Excited-State Behavior of trans and cis Isomers of Stilbene and Stiff Stilbene: A TD-DFT Study. J. Phys. Chem. A 2005, 109, 10058-10067.

(26) Minezawa, N.; Gordon, M. S. Photoisomerization of Stilbene: A Spin-Flip Density Functional Theory Approach. J. Phys. Chem. A 2011, 115, 7901-7911.

(27) Ioffe, I. N.; Granovsky, A. A. Photoisomerization of Stilbene: The Detailed XMCQDPT2 Treatment. J. Chem. Theory Comput. 2013, 9, 4973-4990.

(28) Hohlneicher, G.; Dick, B. Experimental Determination of the Low-Lying Excited A States of trans-Stilbene. J. Photochem. 1984, 27, 215-231.

(29) Anderson, R. J. M.; Holtom, G. R.; Mcclain, W. M. Absolute Two-Photon Absorptivity of trans-Stilbene Near the Two-Photon Absorption Maximum via Three Wave Mixing. J. Chem. Phys. 1977, 66, 3832-3833.

(30) Stachelek, T. M.; Pazoha, T. A.; Mcclain, W. M.; Drucker, R. P. Detection and Assignment of the "Phantom" Photochemical Singlet of trans-Stilbene by Two-Photon Excitation. J. Chem. Phys. 1977, 66, 4540-4543.

(31) Fuke, K.; Sakamoto, S.; Ueda, M.; Itoh, M. Two-Photon Absorption Spectrum of transStilbene: trans-cis Photoisomerization via Upper ${ }^{1} \mathrm{~A}_{\mathrm{g}}$ State. Chem. Phys. Lett. 1980, 74, 546-548. 
Dick, B.; Gonska, H.; Hohlneicher, G. Two Photon Spectroscopy of Dipole Forbidden Transitions III. Experimental Determination of Two Photon Absorption Spectra Including Polarization Control. Ber. Bunsenges. Phys. Chem. 1981, 85, 746-754.

(33) Gudipati, M. S.; Maus, M.; Daverkausen, J.; Hohlneicher, G. Higher Excited States of Aromatic Hydrocarbons. III. Assigning the in-Plane Polarized Transitions of Low-Symmetry Molecules: Chrysene and E-Stilbene. Chem. Phys. 1995, 192, 37-47.

(34) Hohlneicher, G.; Wrzal, R.; Lenoir, D.; Frank, R. Two-Photon Spectra of Stiff Stilbenes: A Contribution to the Assignment of the Low Lying Electronically Excited States of the Stilbene System. J. Phys. Chem. A 1999, 103, 8969-8975.

(35) Heflin, J. R.; Wong, K. Y.; Zamani-Khamiri, O.; Garito, A. F. Nonlinear Optical Properties of Linear Chains and Electron-Correlation Effects. Phys. Rev. B 1988, 38, 1573-1576. (36) Miyazawa, T.; Koshihara, S. Y.; Segawa, Y.; Kira, M. Selective Isomerization of cisStilbene by Non-Resonant Two-Photon Excitation. Chem. Lett. 1995, 24, 217-218.

(37) Miyazawa, T.; Liu, Z.; Liu, C.; Koshihara, S.-Y.; Kira, M. Action Spectra of NonResonant Two-Photon (NRTP) Isomerization of $\alpha, \omega$-Diphenylpolyenes. Chem. Lett. 1996, 25, 1023-1024.

(38) Miyazawa, T.; Liu, C.; Koshihara, S.-Y.; Kira, M. Remarkable Suppression of [2+2] Cycloaddition during Nonresonant Two-photon Photoreaction of trans-Stilbene in the Presence of Tetramethylethylene. Photochem. Photobiol. 1997, 66, 566-568.

(39) Bao, J.; Minitti, M. P.; Weber, P. M. Ring-Closing and Dehydrogenation Reactions of Highly Excited cis-Stilbene: Ultrafast Spectroscopy and Structural Dynamics. J. Phys. Chem. A 2011, $115,1508-1515$.

(40) Elles, C. G.; Rivera, C. A.; Zhang, Y.; Pieniazek, P. A.; Bradforth, S. E. Electronic Structure of Liquid Water from Polarization-Dependent Two-Photon Absorption Spectroscopy. J. Chem. Phys. 2009, 130, 084501. 
(41) Negres, R. A.; Hales, J. M.; Kobyakov, A.; Hagan, D. J.; Van Stryland, E. W. TwoPhoton Spectroscopy and Analysis with a White-Light Continuum Probe. Opt. Lett. 2002, 27, $270-272$.

(42) Negres, R. A.; Hales, J. M.; Hagan, D. J.; Van Stryland, E. W. Experiment and Analysis of Two-Photon Absorption Spectroscopy using a White-Light Continuum Probe. IEEE J. Quantum Elect. 2002, 38, 1205-1216.

(43) Yamaguchi, S.; Tahara, T. Two-Photon Absorption Spectrum of all-trans Retinal. Chem. Phys. Lett. 2003, 376, 237-243.

(44) Yamaguchi, S.; Tahara, T. Observation of an Optically Forbidden State of $\mathrm{C}_{60}$ by Nondegenerate Two-Photon Absorption Spectroscopy. Chem. Phys. Lett. 2004, 390, 136-139.

(45) Nagura, C.; Suda, A.; Kawano, H.; Obara, M.; Midorikawa, K. Generation and Characterization of Ultrafast White-Light Continuum in Condensed Media. Appl. Opt. 2002, 41, 3735-3742.

(46) Buchvarov, I.; Trifonov, A.; Fiebig, T. Toward an Understanding of White-Light Generation in Cubic Media-Polarization Properties Across the Entire Spectral Range. Opt. Lett. 2007, 32, 1539-1541.

(47) Kovalenko, S. A.; Dobryakov, A. L.; Ruthmann, J.; Ernsting, N. P. Femtosecond Spectroscopy of Condensed Phases with Chirped Supercontinuum Probing. Phys. Rev.A 1999, $59,2369-2384$.

(48) Suzuki, H. Relations between Electronic Absorption Spectra and Spatial Configurations of Conjugated Systems. V. Stilbene. Bull. Chem. Soc. Jpn. 1960, 33, 379-388.

(49) Molina, V.; Merchán, M.; Roos, B. O. Theoretical Study of the Electronic Spectrum of trans-Stilbene. J. Phys. Chem. A 1997, 101, 3478-3487.

(50) Angeli, C.; Improta, R.; Santoro, F. On the Controversial Nature of the $1^{1} B_{u}$ and $2^{1} B_{u}$ States of trans-Stilbene: The $n$-Electron Valence State Perturbation Theory Approach. J. Chem. Phys. 2009, 130, 174307. 
(51) Xu, C.; Webb, W. W. Measurement of Two-Photon Excitation Cross Sections of Molecular Fluorophores with Data from 690 to 1050 nm. J. Opt. Soc. Am. B 1996, 13, 481-491.

(52) Kleinschmidt, J.; Rentsch, S.; Tottleben, W.; Wilhelmi, B. Measurement of Strong Nonlinear Absorption in Stilbene-Chloroform Solutions, Explained by Superposition of TwoPhoton Absorption and One-Photon Absorption from the Excited-State. Chem. Phys. Lett. 1974, $24,133-135$.

(53) Anderson, R. J. M.; Holtom, G. R.; Mcclain, W. M. Two-Photon Absorptivities of the alltrans $\alpha, \omega$-Diphenylpolyenes From Stilbene to Diphenyloctatetraene via Three Wave Mixing. $J$. Chem. Phys. 1979, 70, 4310-4315.

(54) Chen, C. H.; Mccann, M. P. Measurements of Two-Photon Absorption Cross Sections of Common Blue Dyes. Opt. Commun. 1987, 63, 335-338.

(55) Svetlichnyi, V. A.; Meshalkin, Y. P. Two-Photon Absorption and Laser Photolysis of trans-Stilbene Substitutes. Opt. Commun. 2007, 280, 379-386.

(56) Monson, P. R.; Mcclain, W. M. Polarization Dependence of the Two-Photon Absorption of Tumbling Molecules with Application to Liquid 1-Chloronaphthalene and Benzene. J. Chem. Phys. 1970, 53, 29-37.

(57) Mcclain, W. M. Excited State Symmetry Assignment Through Polarized Two-Photon Absorption Studies of Fluids. J. Chem. Phys. 1971, 55, 2789-2796.

(58) Monson, P. R.; Mcclain, W. M. Complete Polarization Study of the Two-Photon Absorption of Liquid 1-Chloronaphthalene. J. Chem. Phys. 1972, 56, 4817-4825.

(59) Kwasniewski, S. P.; Deleuze, M. S.; François, J. P. Optical Properties of trans-Stilbene using Semiempirical and Time-Dependent Density Functional Theory: A Comparative Study. Int. J. Quantum Chem. 2000, 80, 672-680.

(60) Greene, B. I.; Hochstrasser, R. M.; Weisman, R. B. Spectroscopic Study of the Picosecond Photoisomerization of Stilbene. Chem. Phys. Lett. 1979, 62, 427-430.

(61) Doany, F. E.; Greene, B. I.; Hochstrasser, R. M. Excitation Energy Effects in the Photophysics of trans-Stilbene in Solution. Chem. Phys. Lett. 1980, 75, 206-208. 
Quick, M.; Berndt, F.; Dobryakov, A. L.; Ioffe, I. N.; Granovsky, A. A.; Knie, C.;

Mahrwald, R.; Lenoir, D.; Ernsting, N. P.; Kovalenko, S. A. Photoisomerization Dynamics of Stiff-Stilbene in Solution. J. Phys. Chem. B 2014, 118, 1389-1402.

(63) Weaver, W. L.; Huston, L. A.; Iwata, K.; Gustafson, T. L. Solvent/Solute Interactions Probed by Picosecond Transient Raman Spectroscopy: Mode-Specific Vibrational Dynamics in $\mathrm{S}_{1}$ trans-Stilbene. J. Phys. Chem. 1992, 96, 8956-8961.

(64) Qian, J.; Schultz, S. L.; Bradburn, G. R.; Jean, J. M. Picosecond Resonance Raman Studies of Vibrational Cooling of Electronically Excited trans-Stilbene in Alcohols and Alkanes. J. Phys. Chem. 1993, 97, 10638-10644.

(65) Qian, J.; Schultz, S. L.; Jean, J. M. Observation of Intramolecular Vibrational Redistribution and Vibrational Cooling in $\mathrm{S}_{1}$ trans-Stilbene and 2-Phenylindene in Solution. Chem. Phys. Lett. 1995, 233, 9-15.

(66) Nakabayashi, T.; Okamoto, H.; Tasumi, M. Probe-Wavelength Dependency of Picosecond Anti-Stokes Raman Spectra of trans-Stilbene in the $\mathrm{S}_{1}$ State. J. Phys. Chem. A 1997, 101, 7189-7193.

(67) Nakabayashi, T.; Okamoto, H.; Tasumi, M. Vibrational Relaxation Dynamics of transStilbene in the Lowest Excited Singlet State. Pump and Probe Wavelength Dependencies of the Picosecond Time-Resolved Anti-Stokes Raman Spectrum. J. Phys. Chem. A 1998, 102, 96869695.

(68) Kovalenko, S. A.; Dobryakov, A. L.; Pollak, E.; Ernsting, N. P. Communication: Optical Cooling of trans-Stilbene. J. Chem. Phys. 2013, 139, 011101.

(69) In principle, the linear versus quadratic intensity dependence of the two signals can be used to separate the relative 2PA and ESA contributions.

(70) Soos, Z. G.; Ramasesha, S.; Galvão, D. S.; Etemad, S. Excitation and Relaxation Energies of trans-Stilbene: Confined Singlet, Triplet, and Charged Bipolarons. Phys. Rev. B 1993, $47,1742-1753$. 
(71) Castleton, C. W. M.; Barford, W. Screening and the Quantitative $\pi$-Model Description of the Optical Spectra and Polarizations of Phenyl Based Oligomers. J. Chem. Phys. 2002, 117, 3570-3582.

(72) Chen, P. C.; Chieh, Y. C. Azobenzene and Stilbene: A Computational Study. J. Mol. Struc.-THEOCHEM 2003, 624, 191-200.

(73) Stålring, J.; Gagliardi, L.; Malmqvist, P.-Å.; Lindh, R. A Theoretical Study of the $2^{1} \mathrm{~A}_{\mathrm{g}}$ $1^{1} \mathrm{~A}_{\mathrm{g}}$ Two-Photon Transition and its Vibronic Band in trans-Stilbene. Mol. Phys. 2002, 100, 1791-1796.

(74) Lei, Y.; Yu, L.; Zhou, B.; Zhu, C.; Wen, Z.; Lin, S. H. Landscapes of Four-Enantiomer Conical Intersections for Photoisomerization of Stilbene: CASSCF Calculation. J. Phys. Chem. A 2014, DOI: 10.1021/jp5020109.

(75) Takeuchi, S.; Ruhman, S.; Tsuneda, T.; Chiba, M.; Taketsugu, T.; Tahara, T.

Spectroscopic Tracking of Structural Evolution in Ultrafast Stilbene Photoisomerization. Science 2008, 322, 1073-1077.

(76) Kovalenko, S. A.; Dobryakov, A. L.; Ernsting, N. P. An Efficient Setup for Femtosecond Stimulated Raman Spectroscopy. Rev. Sci. Instrum. 2011, 82, 063102.

(77) Dobryakov, A. L.; Ioffe, I.; Granovsky, A. A.; Ernsting, N. P.; Kovalenko, S. A.

Femtosecond Raman Spectra of cis-Stilbene and trans-Stilbene with Isotopomers in Solution. J. Chem. Phys. 2012, 137, 244505.

(78) Kukura, P.; Mccamant, D. W.; Yoon, S.; Wandschneider, D. B.; Mathies, R. A. Structural Observation of the Primary Isomerization in Vision with Femtosecond-Stimulated Raman.

Science 2005, 310, 1006-1009.

(79) Shim, S.; Dasgupta, J.; Mathies, R. A. Femtosecond Time-Resolved Stimulated Raman Reveals the Birth of Bacteriorhodopsin's J and K Intermediates. J. Am. Chem. Soc. 2009, 131, $7592-7597$. 
(80) Hoffman, D. P.; Mathies, R. A. Photoexcited Structural Dynamics of an Azobenzene Analog 4-nitro-4'-dimethylamino-azobenzene from Femtosecond Stimulated Raman. Phys. Chem. Chem. Phys. 2012, 14, 6298-6306.

(81) Hoffman, D. P.; Ellis, S. R.; Mathies, R. A. Characterization of a Conical Intersection in a Charge-Transfer Dimer with Two-Dimensional Time-Resolved Stimulated Raman Spectroscopy. J. Phys. Chem. A 2014, 118, 4955-4965.

(82) Pontecorvo, E.; Ferrante, C.; Elles, C. G.; Scopigno, T. Structural Rearrangement Accompanying the Ultrafast Electrocyclization Reaction of a Photochromic Molecular Switch. $J$. Phys. Chem. B 2014, 118, 6915-6921. 


\section{TOC IMAGE}

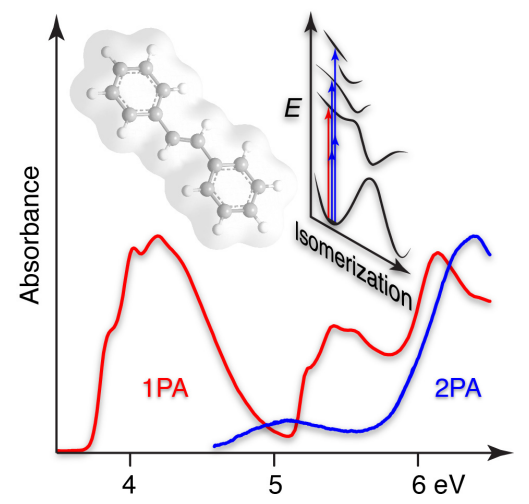

